\title{
Development of an Ion-Pairing Reagent and HPLC-UV Method for the Detection and Quantification of Six Water-Soluble Vitamins in Animal Feed
}

\author{
Ho Jin Kim \\ National Agricultural Products Quality Management Service, Gimcheon 740-871, Republic of Korea \\ Correspondence should be addressed to Ho Jin Kim; rex7878@korea.kr
}

Received 23 May 2016; Revised 14 June 2016; Accepted 15 June 2016

Academic Editor: Josep Esteve-Romero

Copyright (C) 2016 Ho Jin Kim. This is an open access article distributed under the Creative Commons Attribution License, which permits unrestricted use, distribution, and reproduction in any medium, provided the original work is properly cited.

\begin{abstract}
A novel and simple method for detecting six water-soluble vitamins in animal feed using high performance liquid chromatography equipped with a photodiode array detector (HPLC/PDA) and ion-pairing reagent was developed. The chromatographic peaks of the six water-soluble vitamins were successfully identified by comparing their retention times and UV spectra with reference standards. The mobile phase was composed of buffers A ( 5 mM PICB- 6 in $0.1 \% \mathrm{CH}_{3} \mathrm{COOH}$ ) and B (5 mM PICB-6 in $65 \%$ methanol). All peaks were detected using a wavelength of $270 \mathrm{~nm}$. Method validation was performed in terms of linearity, sensitivity, selectivity, accuracy, and precision. The limits of detection (LODs) for the instrument employed in these experiments ranged from 25 to $197 \mu \mathrm{g} / \mathrm{kg}$, and the limits of quantification (LOQs) ranged from 84 to $658 \mu \mathrm{g} / \mathrm{kg}$. Average recoveries of the six water-soluble vitamins ranged from $82.3 \%$ to $98.9 \%$. Method replication resulted in intraday and interday peak area variation of $<5.6 \%$. The developed method was specific and reliable and is therefore suitable for the routine analysis of water-soluble vitamins in animal feed.
\end{abstract}

\section{Introduction}

Vitamins are essential for nutrition and physiological function, and therefore, their intake is necessary for humans and animals, as the body is unable to synthesize these essential nutrients required for growth [1]. Vitamins can be classified into water-soluble and fat-soluble vitamins; the former includes niacin, nicotinamide, vitamin B6, vitamin $\mathrm{B} 2$, vitamin $\mathrm{B} 1$, folic acid, pantothenic acid, and vitamin $\mathrm{C}$, while the latter includes vitamin $\mathrm{A}$, vitamin $\mathrm{D}$, vitamin $\mathrm{E}$, and vitamin K [2]. Vitamins have specific and important functions, and as such, both humans and animals require vitamins to achieve and maintain health and productivity [3]. Vitamins function to control various endogenous metabolic activities taking place in the body, such as energy and amino acid metabolism [4]. Although only small amounts of vitamins are required, vitamin deficiency (or indeed, excess) can lead to diseases such as beriberi [5] and dermatitis [2, 6, 7]. Thus, to prevent a vitamin deficiency or excess in animals, as well as to attain maximum performance, the control of feed additives is necessary [8]. Indeed, vitamins are essential for maintaining normal metabolic processes in animals, while also retaining their condition and performance. As animals are unable to synthesize vitamins, the small amounts of these nutrients required must be supplied in their feed [9].

Various methods have been reported for the characterization of water-soluble vitamins, such as high performance liquid chromatography (HPLC) coupled with ultraviolet (UV) detection [10, 11], liquid chromatography (LC) coupled with mass spectrometry (MS) [12], LC coupled with MS/MS [13], and gas chromatography [14]. The most commonly used methods for the determination of vitamin components are based on HPLC separation. LC-MS and LC-MS/MS, which provide information about the molecular mass and structural features of components, are considered more useful than other methods for the separation, identification, and quantification of the characteristic vitamin compounds. However, since these methods are expensive to purchase and maintain, many laboratories prefer HPLC-UV detection [15], which is less costly, relatively convenient to operate, and suitable for 
<smiles>NC(=O)c1cccnc1</smiles>

Folic acid<smiles>Cc1ncc(CO)c(CO)c1O</smiles>

Pyridoxine (vit B6)<smiles>Cc1cc2nc3c(=O)[nH]c(=O)nc-3n(C[C@H](O)[C@H](O)CCO)c2cc1C</smiles>

Riboflavin<smiles>Cc1ncc(C[n+]2csc(CCO)c2C)c(N)n1</smiles>

Thiamine (vit B1)

FIGURE 1: Chemical structures of six water-soluble vitamins analyzed in this study.

the routine analysis of vitamins. Ion-paring reagent we used in this study has amine group, so it is suitable for watersoluble vitamins analysis as well as iodine speciation analysis and pesticide residue analysis [16-18].

In this study, six water-soluble vitamins in animal feed (nicotinic acid (niacin), nicotinamide, folic acid, riboflavin, pyridoxine (vitamin $\mathrm{B} 6$ ), and thiamine (vitamin B1)) (Figure 1) were analyzed simultaneously. Complete animal feeds have complex matrixes containing fat, protein, carbohydrate, salt, and so on. Water-soluble vitamins we targeted have amine functional group which represents polarity. To increase the selectivity of the water-soluble vitamins in complicated matrixes, these nontarget matrixes should be removed. In addition, simultaneous analytical method for the determination of 6 water-soluble vitamins has been rarely reported. Moreover, AOAC method or other international methods each have vitamin method. Therefore, this newly developed simultaneous method should be validated in various feed matrixes. A novel analytic method is therefore reported, which is suitable for detecting and quantifying the vitamins present in animal feed, while also aiding in the management of feed standards.

\section{Experimental}

2.1. Samples. The water-soluble vitamins present in animal feed were targeted in this study. Animal feed samples were purchased from a local supermarket. A total of 30 animal feed samples were used, and 20 dried samples were pulverized into fine powders (HMF-100, HANIL Electric Co., Seoul, Korea). The pulverizer was set at a maximum speed of $22,000 \mathrm{rpm}$ to give fine powders ranging in size from 400 to $1000 \mu \mathrm{m}$. The remaining 10 liquefied samples were used as received. All samples were stored at $4^{\circ} \mathrm{C}$.

2.2. Chemicals and Reagents. HPLC grade acetonitrile, methanol, and acetic acid were purchased from Merck (Darmstadt, Germany), and PICB-6 (PIC, paired-ion chromatography; B, separate bases) was purchased from Waters (St. Milford, MA, USA). Water was purified using a Milli-Q ${ }^{\circledR}$ $\mathrm{RiOs}^{\mathrm{TM}} / \mathrm{Elix}^{\circledR}$ water purification system (Millipore, Bedford, MA, USA). Nicotinic acid (niacin), nicotinamide, pyridoxine (vitamin B6), riboflavin, thiamine (vitamin B1), and folic acid standards were purchased from Sigma Aldrich Chemie $\mathrm{GmbH}$ (Bellefonte, PA, USA). All other chemicals and solvents were of reagent grade or higher.

2.3. Standard Preparation. The six vitamins were classified into three groups, groups 1, 2, and 3, and stock solutions were prepared using the preprocessing reagent, water, and $0.01 \mathrm{~N}$ $\mathrm{NaOH}$ to obtain a final vitamin concentration of $100 \mathrm{mg} / \mathrm{L}$. Group 1 consisted of thiamine and pyridoxine, and the stock solutions were prepared by mixing the standard preparation 
with water $(100 \mathrm{~mL})$. Group 2 consisted of riboflavin and folic acid and was prepared by mixing the standard preparation $(10 \mathrm{mg}), 0.01 \mathrm{~N} \mathrm{NaOH}$, and water $(100 \mathrm{~mL})$. Group 3 consisted of nicotinic acid and nicotinamide and was prepared by mixing the standard preparation $(10 \mathrm{mg})$ with the preprocessing reagent $(100 \mathrm{~mL})$. Working solutions were prepared by diluting the stock solutions, and all solutions were refrigerated.

2.4. Sample Preparation. The samples were prepared for extraction by the ion-pairing reagent. Each homogenized sample from animal feed ( $1.5 \mathrm{~g})$ was placed into a centrifuge tube and mixed with the extraction solvent $(10 \mathrm{~mL}, 5 \mathrm{mM}$ PICB-6 in $\left.0.1 \% \mathrm{CH}_{3} \mathrm{COOH}\right)$. A portion of the liquefied sample (10 g) was added to a $25 \mathrm{~mL}$ volumetric flask, and the volume was made up to $25 \mathrm{~mL}$ using the extraction solvent (5 mM PICB-6 in 0.1\% $\mathrm{CH}_{3} \mathrm{COOH}$ ). We used PICB-6 as ion-pairing reagent because most of vitamins we determined showed higher resolution when we used PICB-6 than other ion-pairing reagents. The mixtures were then homogenized for $10 \mathrm{~min}$, sonicated for $10 \mathrm{~min}$ at room temperature, and centrifuged at $13,000 \mathrm{rpm}$ for $10 \mathrm{~min}$ at $4^{\circ} \mathrm{C}$. The resulting supernatant was filtered through a $0.45 \mu \mathrm{m}$ disposable filter (Whatman). All measurements were performed in triplicate. Finally, the stabilities of the standard preparations were determined over one week in an amber vial at room temperature, with samples being taken for analysis every $24 \mathrm{~h}$.

2.5. HPLC Analysis. HPLC was performed on a Shiseido Nanospace SI-2 system (Shiseido, Tokyo, Japan) equipped with a binary solvent delivery pump, an autosampler, and a photodiode array detector (PDA) and was controlled using the EZChrome Elite software (Agilent Technologies, Palo Alto, CA, USA). A reversed phase Unison UK-C18 $(100 \mathrm{~m}$ $\times 4.6 \mathrm{~mm}, 3 \mu \mathrm{m}$ particle size) (Tokyo, Japan) column was used for all separations at a column temperature of $40^{\circ} \mathrm{C}$. Prior to use, the mobile phase was filtered through a $0.45 \mu \mathrm{m}$ membrane filter (Millipore, Milford, MA, USA) and degassed under vacuum. The mobile phase was composed of buffers A (5 mM PICB-6 in 0.1\% $\mathrm{CH}_{3} \mathrm{COOH}$ ) and B (5 mM PICB-6 in $65 \%$ methanol) with the following gradient elution: $0 \mathrm{~min}$, $10 \% \mathrm{~B} ; 0-2 \mathrm{~min}, 10 \% \mathrm{~B} ; 2-22 \mathrm{~min}, 70 \% \mathrm{~B} ; 22-27 \mathrm{~min}, 70 \% \mathrm{~B}$; 27-28 $\mathrm{min}, 10 \% \mathrm{~B}$; and $28-35 \mathrm{~min}, 10 \% \mathrm{~B}$. The sample injection volume was $2 \mu \mathrm{L}$, and the flow rate was set at $0.5 \mathrm{~mL} / \mathrm{min}$. All peaks were detected using a wavelength of $270 \mathrm{~nm}$.

2.6. Method Validation. Method validation was performed according to the guidelines set by the International Conference on Harmonization ( $\mathrm{ICH}, 2005)$ [18] and the International Union of Pure and Applied Chemistry (IUPAC, 2002) [19]. The method was validated for linearity, sensitivity, selectivity, accuracy, and precision, as outlined in Figure 2 and Table 1.

\section{Results and Discussion}

3.1. Method Validation. The developed RP-HPLC/UV method incorporating an ion-pairing reagent was validated

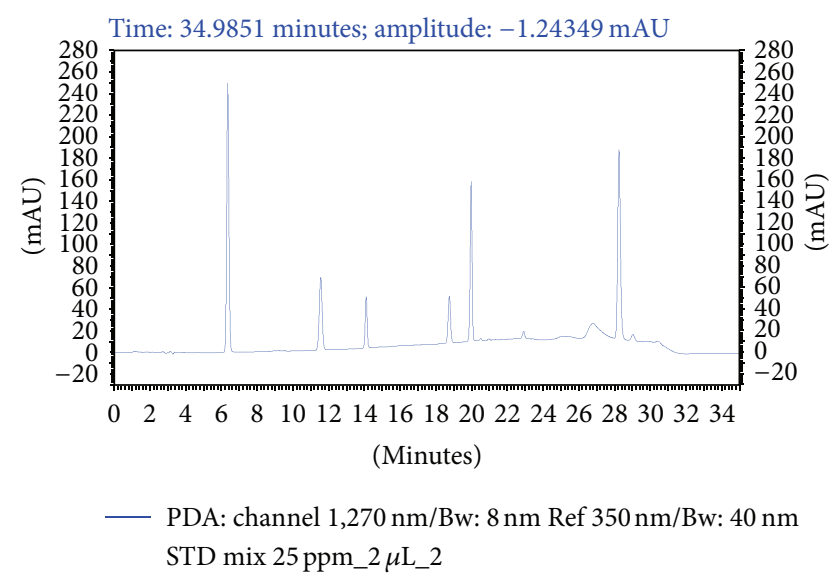

FIGURE 2: HPLC chromatograms of a mixture of six vitamins detected at $270 \mathrm{~nm}$. (1) Nicotinic acid, (2) nicotinamide, (3) pyridoxine, (4) folic acid, (5) riboflavin, and (6) thiamin.

to verify that its performance met the requirements for routine vitamin analysis. Several performance characteristics were measured, including selectivity, linearity, sensitivity, accuracy, and precision.

Selectivity was determined by the absence of interference in the chromatographic window, and this was measured using blank chromatograms at the specific quantification wavelength (i.e., $270 \mathrm{~nm}$ ). As shown in Figure 2 and Table 1, the chromatograms of the water-soluble vitamins indicate successful separation of all six compounds in $<29 \mathrm{~min}$, with good resolution (5.55-34.85) and asymmetry (0.94-1.08), thereby indicating satisfactory selectivity for this HPLC system.

Linearity was assessed by building external calibration curves for each compound using the vitamin-containing working solutions. Calibration curves were obtained by plotting the analyte peak area versus its concentration over seven different concentrations. Each concentration of the mixed standard solution was injected in triplicate, and then, the regression parameters were calculated. The results are shown in Table 1. Correlation coefficients $\left(r^{2}>0.999\right)$ were obtained for all compounds studied. These results demonstrate that an external standard calibration can be applied for quantitative purposes.

The sensitivity of the developed method was evaluated by determining the LOD and LOQ values. Under the described chromatographic conditions, these values were calculated based on the response and slope of each regression equation at signal-to-noise ratios $(S / N)$ of $3: 1$ and $10: 1$, respectively. For the different components, the LOD values ranged from 25 to $197 \mu \mathrm{g} / \mathrm{kg}$, while the LOQ values ranged from 84 to $658 \mu \mathrm{g} / \mathrm{kg}$. Detailed data are shown in Table 1.

The method precision was then determined by measuring the intra- and interday precision. For the intraday precision, six replicates of the mixed standard solutions were analyzed within $1 \mathrm{~d}$, while the solutions were examined in triplicate for three consecutive days for the intraday precision. The precision was expressed as the percentage of the relative standard deviation (\% RSD). The overall intraday $\% \mathrm{RSD}$ values were $<5.6 \%$, while the interday values were $<4.8 \%$ (see Table 1 ). 


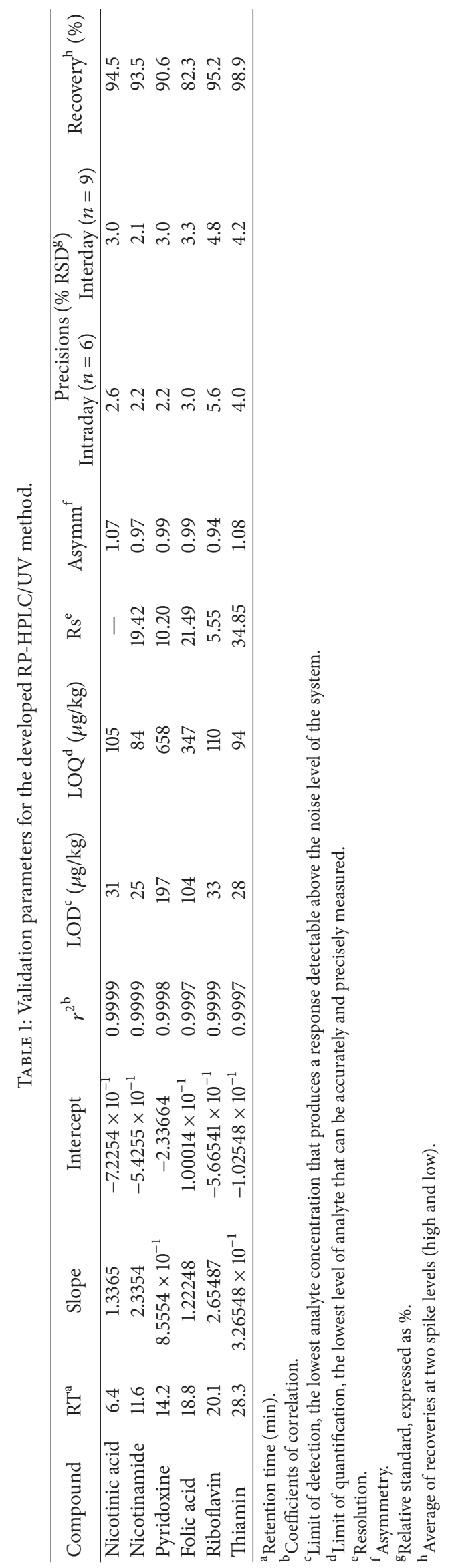


TABLE 2: Stability of six water-soluble vitamins.

\begin{tabular}{lcccccc}
\hline Storage time $(\mathrm{d})$ & Nicotinic acid & Nicotinamide & Folic acid & Pyridoxine & Riboflavin & Thiamine \\
\hline 1 & $98.70 \pm 0.05$ & $97.54 \pm 0.14$ & $99.83 \pm 0.21$ & $98.58 \pm 0.02$ & $97.66 \pm 1.10$ & $99.24 \pm 0.20$ \\
2 & $96.57 \pm 0.03$ & $96.03 \pm 0.02$ & $89.01 \pm 0.02$ & $96.12 \pm 0.54$ & $95.69 \pm 0.52$ & $97.10 \pm 0.66$ \\
3 & $94.29 \pm 0.12$ & $92.12 \pm 0.24$ & $63.38 \pm 0.05$ & $93.03 \pm 0.86$ & $94.44 \pm 0.99$ & $95.93 \pm 1.65$ \\
4 & $85.32 \pm 0.08$ & $83.66 \pm 1.03$ & $60.93 \pm 1.21$ & $84.42 \pm 0.39$ & $84.93 \pm 1.63$ & $87.18 \pm 1.87$ \\
5 & $70.85 \pm 0.07$ & $73.25 \pm 0.08$ & $56.24 \pm 0.34$ & $73.15 \pm 1.09$ & $71.03 \pm 0.83$ & $72.93 \pm 0.39$ \\
6 & $63.03 \pm 0.16$ & $67.26 \pm 0.12$ & $52.62 \pm 0.44$ & $65.05 \pm 1.21$ & $66.38 \pm 0.41$ & $62.01 \pm 0.33$ \\
7 & $59.63 \pm 0.13$ & $59.90 \pm 1.08$ & $49.59 \pm 0.20$ & $60.99 \pm 0.50$ & $58.99 \pm 0.62$ & $59.56 \pm 0.72$ \\
\hline
\end{tabular}

TABLE 3: Determination of the vitamin contents $(\mathrm{mg} / \mathrm{kg})$ in animal feed using the proposed method.

\begin{tabular}{|c|c|c|c|c|c|c|}
\hline Sample & Nicotinic acid & Nicotinamide & Folic acid & Pyridoxine & Riboflavin & Thiamine \\
\hline 1 & 5,231 & 6,380 & - & 20,449 & - & 10,648 \\
\hline 2 & 1,868 & 42,085 & 102 & - & - & 9,803 \\
\hline 3 & 20,009 & - & - & 9,027 & 4,093 & - \\
\hline 4 & 46,289 & - & 452 & - & 29,344 & 8,014 \\
\hline 5 & 9,227 & 8,263 & - & 10,382 & 30,298 & - \\
\hline 6 & 19,263 & - & 780 & 37,030 & - & 5,226 \\
\hline 7 & - & 49,920 & - & 2,009 & - & 5,814 \\
\hline 8 & - & 2,892 & 358 & - & 10,023 & 20,687 \\
\hline 9 & 16,354 & 34,893 & - & 5,934 & 3,015 & - \\
\hline 10 & 8,308 & - & 552 & - & 23,589 & - \\
\hline 11 & 5,360 & 7,099 & 837 & - & 24,263 & 18,893 \\
\hline 12 & - & 10,298 & 960 & 9,982 & - & 4,300 \\
\hline 13 & 6,993 & 26,407 & 200 & 6,057 & 10,360 & - \\
\hline 14 & 21,960 & 48,062 & - & 2,918 & 5,430 & 9,366 \\
\hline 15 & - & - & 753 & - & - & 48,930 \\
\hline 16 & - & 6,337 & 200 & 49,530 & - & 1,380 \\
\hline 17 & 40,826 & 12,086 & - & - & 5,669 & 4,085 \\
\hline 18 & 3,698 & - & 901 & 8,006 & 7,802 & 30,569 \\
\hline 19 & - & 1,339 & 5,012 & - & 9,829 & - \\
\hline 20 & - & - & 303 & 39,807 & 4,369 & - \\
\hline 21 & 30,158 & 2,580 & - & 5,980 & - & 10,230 \\
\hline 22 & 4,803 & - & 807 & - & 36,820 & 5,628 \\
\hline 23 & 10,009 & - & 650 & 48,301 & - & 7,083 \\
\hline 24 & - & 15,369 & - & 2,065 & & 8,292 \\
\hline 25 & 25,801 & - & - & - & 6,043 & - \\
\hline 26 & 26,990 & - & 268 & - & 8,823 & 1,359 \\
\hline 27 & 9,045 & - & - & 4,6933 & - & 4,860 \\
\hline 28 & 4,960 & 9,963 & - & - & - & - \\
\hline 29 & - & 2,860 & - & 10,297 & 6,099 & - \\
\hline 30 & 18,203 & 5,802 & 692 & 4,950 & - & 20,560 \\
\hline
\end{tabular}

The accuracy was evaluated by adding the mixed standard solutions at two different concentrations (high: $20.0 \mathrm{mg} / \mathrm{kg}$; low: $2.0 \mathrm{mg} / \mathrm{kg}$ ) to feed from the Association of American Feed Control Official (AFFCO, 201591-Swine Mineral and Vitamin Supplement). The mixture was extracted using the developed ion-pairing reagent technique and HPLC method. All tests were performed in triplicate. Excellent recovery rates of $82.3-98.9 \%$ indicated a high level of accuracy for this method, as detailed in Table 1.
The stability of all standard solutions was also tested over $7 \mathrm{~d}$, with the majority having decomposed within $3 \mathrm{~d}$. In particular, in the case of folic acid, rapid decomposition was observed after $3 \mathrm{~d}$ compared to that of the other water-soluble vitamins (i.e., 63.38\% cf. 92.12-95.93\%). These results demonstrated that it is necessary to prepare a fresh standard preparation for each analysis. Detailed results are shown in Table 2.

Based on the above validation data, the proposed method was concluded to provide good linearity, sensitivity, 
selectivity, accuracy, and precision for the simultaneous analysis of water-soluble vitamins.

3.2. Application of the Developed Method. Among the 30 different animal feeds that were analyzed in this study, vitamins were detected in all animal feeds purchased from supermarkets (Table 3). Each sample was analyzed in triplicate. Identification of the six compounds was by comparison of their retention times and UV spectra with those of the standards and the pure compounds. The qualitative and quantitative compositions of the six compounds in the animal feed varied significantly. More specifically, the nicotinic acid content ranged from 1,868 to $46,289 \mathrm{mg} / \mathrm{kg}$ with an average content of $15,969 \mathrm{mg} / \mathrm{kg}$, while the nicotinamide content ranged from 1,339 to $49,920 \mathrm{mg} / \mathrm{kg}$ with an average of $16,257 \mathrm{mg} / \mathrm{kg}$. In addition, the content of folic acid varied between 102 and $5,012 \mathrm{mg} / \mathrm{kg}$ (average $=813 \mathrm{mg} / \mathrm{kg}$ ), while that of pyridoxine was between 2,065 and $49,530 \mathrm{mg} / \mathrm{kg}$ (average $=17,758 \mathrm{mg} / \mathrm{kg}$ ). Finally, the riboflavin and thiamine contents ranged from 3,015 to $36,820 \mathrm{mg} / \mathrm{kg}$ (average = $13,286 \mathrm{mg} / \mathrm{kg}$ ) and from 1,359 to $48,930 \mathrm{mg} / \mathrm{kg}$ (average = $11,786 \mathrm{mg} / \mathrm{kg}$ ), respectively.

Thus, a simple, qualitative, and quantitative method for the simultaneous detection and quantification of six vitamin compounds from animal feed was successfully developed and validated using RP-HPLC/UV detection. Furthermore, the extraction process was optimized using an ion-pairing reagent. The proposed method showed accuracy and precision and was successfully employed for analyzing different types of feeds. Analytical results demonstrated that this HPLC method provides a good alternative for routine analysis, owing to its simplicity, specificity, and sensitivity. Finally, it exhibits the potential to be applied as a reliable quality evaluation method for animal feed.

\section{Competing Interests}

The author declares that there are no competing interests regarding the publication of this paper.

\section{References}

[1] S. Poongothai, R. Ilavarasan, and C. M. Karrunakaran, "Simultaneous and accurate determination of vitamins B1, B6, B12 and alpha-lipoic acid in multivitamin capsule by reverse-phase high performance liquid chromatographic method," International Journal of Pharmacy and Pharmaceutical Sciences, vol. 2, no. 4, pp. 133-139, 2010.

[2] P. Moreno and V. Salvadó, "Determination of eight water- and fat-soluble vitamins in multi-vitamin pharmaceutical formulations by high-performance liquid chromatography," Journal of Chromatography A, vol. 870, no. 1-2, pp. 207-215, 2000.

[3] "Nutrient requirements of beef cattle in Indochinese Peninsula," in Chapter 8: Feed Additives Supplementation in Beef Cattle, pp. 73-79, Japan International Research Center for Agricultural Sciences (JIRCAS), Tsukuba City, Japan, 2010.

[4] B. Weiss, "Update on vitamin nutrition of dairy cows," in Proceedings of the Ruminant Health and Nutrition Conference, Syracuse, NY and New England Dairy Feed Conference, West Lebanon, NH, USA, March 2005.
[5] K. Sriram, W. Manzanares, and K. Joseph, "Thiamine in nutrition therapy," Nutrition in Clinical Practice, vol. 27, no. 1, pp. 4150, 2012.

[6] H. J. Powers, "Riboflavin (vitamin B-2) and health," American Journal of Clinical Nutrition, vol. 77, no. 6, pp. 1352-1360, 2003.

[7] G. F. M. Ball, Vitamins in Foods: Analysis, Bioavailability, and Stability, Taylor \& Francis, New York, NY, USA, 2005.

[8] H. B. Sewell, "Feed additives for beef cattle," Agricultural Publication G02075, University Extension, University of MissouriColimbia, Columbia, Miss, USA, 1993.

[9] N. Alber, G. Gotterbarm, W. Heimbeck, Th. Keller, J. Seehawer, and T. D. Tran, Vitamins in Animal Nutrition, Agrimedia $\mathrm{GmbH}, 2002$.

[10] D. Ivanović, A. Popović, D. Radulović, and M. Medenica, "Reversed-phase ion-pair HPLC determination of some watersoluble vitamins in pharmaceuticals," Journal of Pharmaceutical and Biomedical Analysis, vol. 18, no. 6, pp. 999-1004, 1999.

[11] C. M. Cho, J. H. Ko, and W. J. Cheong, "Simultaneous determination of water-soluble vitamins excreted in human urine after eating an overdose of vitamin pills by a HPLC method coupled with a solid phase extraction," Talanta, vol. 51, no. 4, pp. 799$806,2000$.

[12] J. E. Oosterink, E. F. G. Naninck, A. Korosi, P. J. Lucassen, J. B. van Goudoever, and H. Schierbeek, "Accurate measurement of the essential micronutrients methionine, homocysteine, vitamins B6, B12, B9 and their metabolites in plasma, brain and maternal milk of mice using LC/MS ion trap analysis," Journal of Chromatography B, vol. 998-999, pp. 106-113, 2015.

[13] C. Jenkinson, A. E. Taylor, Z. K. Hassan-Smith et al., "High throughput LC-MS/MS method for the simultaneous analysis of multiple vitamin D analytes in serum," Journal of Chromatography B, vol. 1014, pp. 56-63, 2016.

[14] M. Du and D. U. Ahn, "Simultaneous analysis of tocopherols, cholesterol, and phytosterols using gas chromatography," Journal of Food Science, vol. 67, no. 5, pp. 1696-1700, 2002.

[15] H. J. Kim, M. H. Jeong, H. J. Park, W. C. Kim, and J. E. Kim, "Development of an immunoaffinity chromatography and HPLC-UV method for determination of 16 sulfonamides in feed," Food Chemistry, vol. 196, pp. 1144-1149, 2016.

[16] J. Sun, D. Wang, H. Cheng, J. Liu, Y. Wang, and Z. Xu, "Use of ion-pairing reagent for improving iodine speciation analysis in seaweed by pressure-driven capillary electrophoresis and ultraviolet detection," Journal of Chromatography A, vol. 1379, pp. 112-117, 2015.

[17] H. Guo, L. S. Riter, C. E. Wujcik, and D. W. Armstrong, "Quantitative analysis of dicamba residues in raw agricultural commodities with the use of ion-pairing reagents in LC-ESIMS/MS," Talanta, vol. 149, pp. 103-109, 2016.

[18] E. Yamamoto, Y. Ishihama, and N. Asakawa, "Application of partially fluorinated carboxylic acids as ion-pairing reagents in LC/ESI-MS," Talanta, vol. 127, pp. 219-224, 2014.

[19] M. Thompson, S. L. R. Ellison, and R. Wood, "Harmonized guidelines for single laboratory validation of methods of analysis," International Union of Pure and Applied Chemistry, vol. 74, pp. 835-855, 2002. 

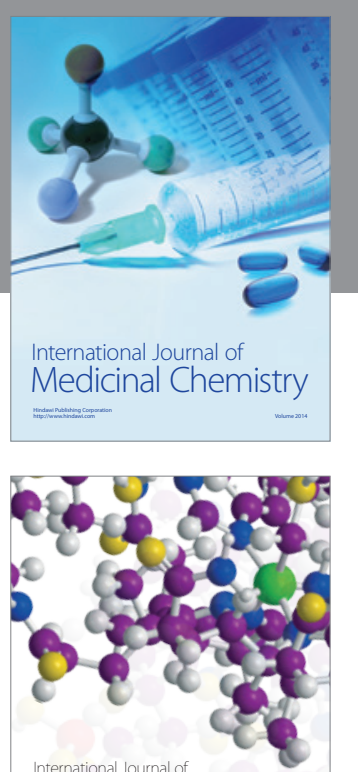

Carbohydrate Chemistry

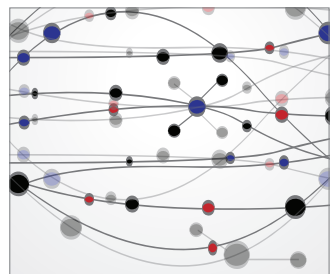

The Scientific World Journal
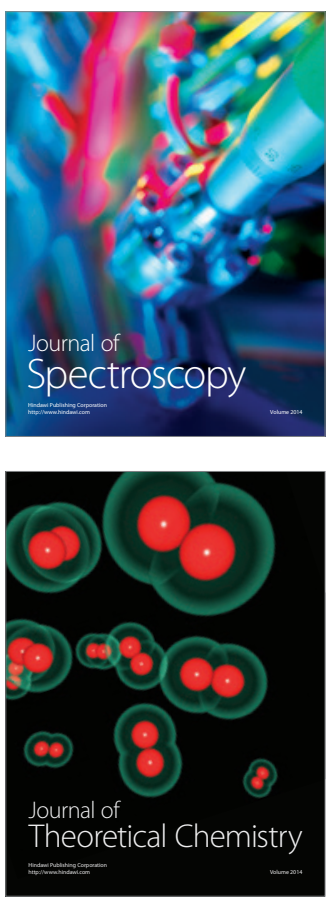
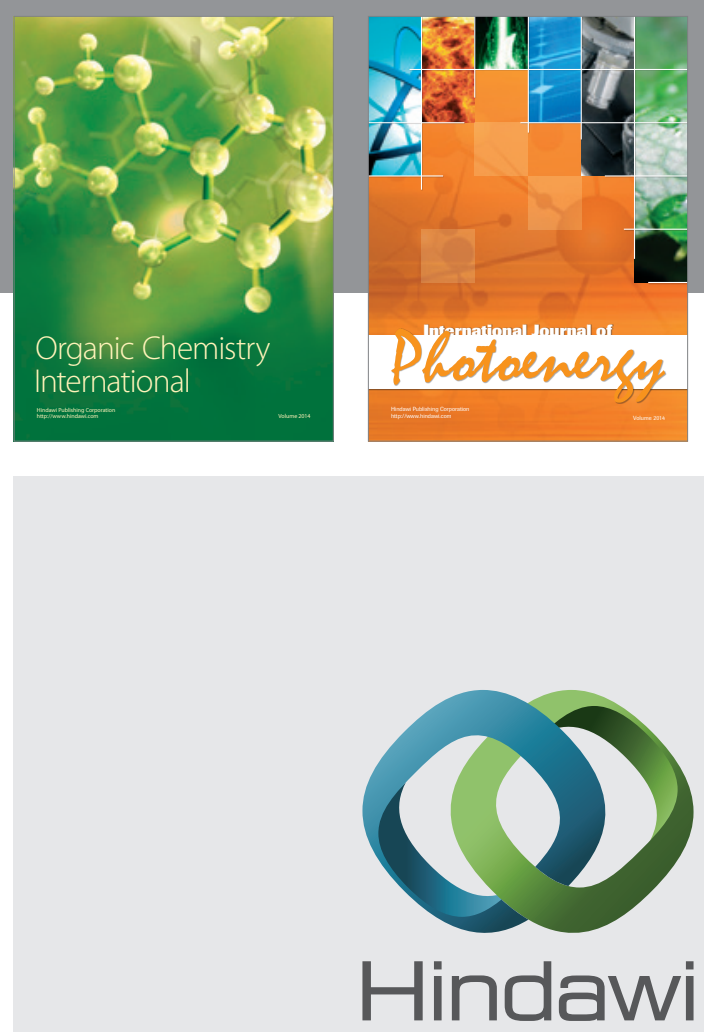

Submit your manuscripts at

http://www.hindawi.com

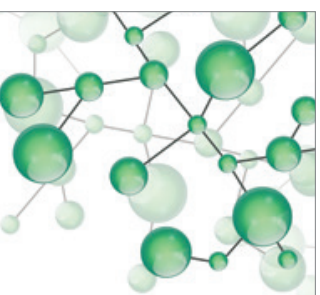

International Journal of

Inorganic Chemistry

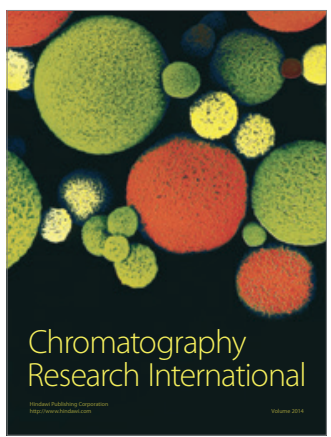

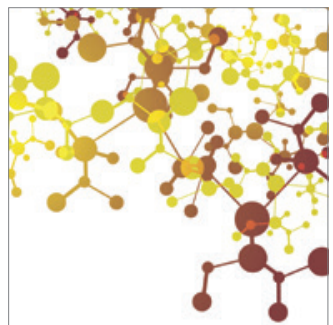

Applied Chemistry
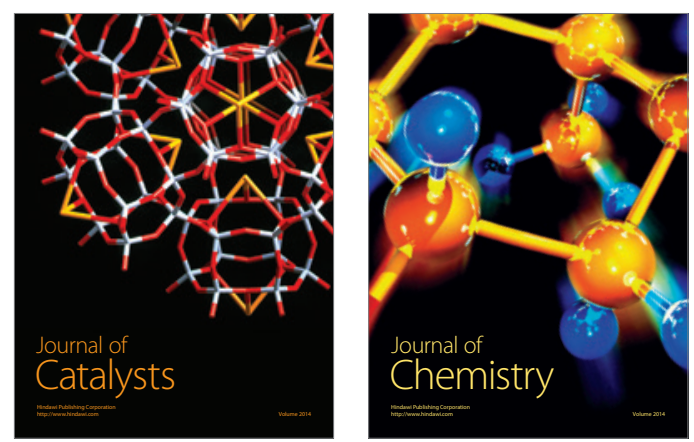
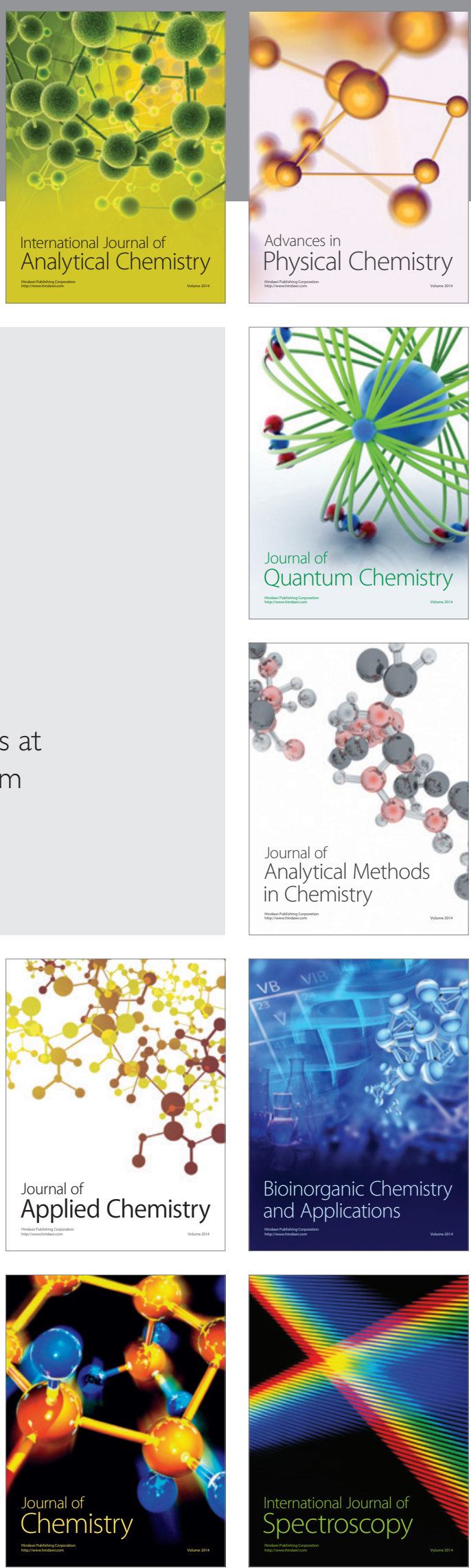